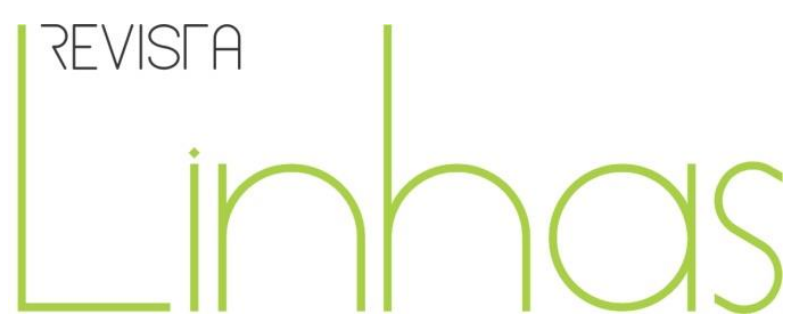

\title{
Acceso y uso de las nuevas tecnologías para aprender matemática desde la perspectiva del estudiante
}

\begin{abstract}
Resumen
El objetivo de este artículo es describir, desde la perspectiva de los estudiantes, su desempeño en la habilidad de acceder y utilizar nuevas tecnologías para aprender matemática. El contexto de la investigación es el Taller Inicial Obligatorio del área de Matemática, una de las primeras asignaturas de la Universidad Nacional General Sarmiento, Argentina. Enmarcamos, teóricamente, el estudio en el Enfoque Cognitivo en Educación Matemática, considerando, en particular, las habilidades matemáticas. Incluimos aquí el diseño de una rúbrica que permite evaluar el acceso y uso de nuevas tecnologías para el aprendizaje de la matemática, considerada como habilidad. Diseñamos y fundamentamos un cuestionario, y lo aplicamos a todos los alumnos del Taller, en el primer trimestre de 2021. Analizamos los datos desde una perspectiva cuantitativa-cualitativa. Esto permite, además de lograr la descripción esperada, proponer una tipología de desempeños, respecto a esta habilidad. Finalmente, expresamos perspectivas que abren este estudio.
\end{abstract}

Palabras clave: Habilidades matemáticas; nuevas tecnologías; software matemático; información matemática; aprendizaje matemático.
Mabel Alicia Rodríguez

Universidad Nacional de General

Sarmiento - Argentina mrodri@campus.ungs.edu.ar

\section{Víctor Hugo González}

Universidad Nacional de General

Sarmiento - Argentina

vgonzalez@campus.ungs.edu.ar

\section{Doris Rodríguez}

Universidad Nacional de General

Sarmiento - Argentina rodriguezdoris.22@gmail.com

\section{Para citar este artigo:}

RODRíGUEZ, Mabel Alicia; GONZÁLEZ, Víctor Hugo; RODRíGUEZ, Doris. Acceso y uso de las nuevas tecnologías para aprender matemática desde la perspectiva del estudiante. Revista Linhas. Florianópolis, v. 22, n. 49, p. 289-319, maio/ago. 2021. 


\section{Access and use of new technologies for learning mathematics, from student's perspective}

\begin{abstract}
The aim of this article is to describe, from the perspective of students, their performance in the skill accessing and using new technologies to learn mathematics. The context of the research is the Initial Obligatory Workshop in Mathematics, one of the first subjects at the National University of General Sarmiento, Argentina. We frame, theoretically, the study in the Cognitive Approach in Mathematics Education, considering, in particular, mathematical skills. We include here the design of a rubric that allows assessing the access and use of new technologies to learn mathematics, considered as a skill. We design a questionnaire, based on the theoretical frame, and we applied to all the students of the Workshop, in the first quarter of 2021. We analyze the data from a quantitativequalitative perspective. This allows, in addition to achieving the expected description, to propose a typology of performances, regarding this ability. Finally, we express perspectives that this study opens.
\end{abstract}

Keywords: Mathematical skills; new technologies; mathematical software; mathematical information; mathematical learning.
Acesso e uso de novas tecnologias para aprender matemática na perspectiva do aluno

\section{Resumo}

O objetivo deste artigo é descrever, na perspectiva dos alunos, seu desempenho na capacidade de acesso e uso de novas tecnologias para aprender matemática. O contexto da pesquisa é o Taller Inicial Obligatorio da área de Matemática, uma das primeiras disciplinas da Universidade Nacional General Sarmiento, Argentina. Enquadramos, teoricamente, $\mathrm{o}$ estudo na Abordagem Cognitiva em Educação Matemática, considerando, em particular, habilidades matemáticas. Incluímos aqui o desenho de uma rubrica que permite avaliar o acesso e o uso de novas tecnologias para aprender matemática, considerada como uma habilidade. Projetamos e baseamos um questionário, baseado no quadro teórico, e aplicamos a todos os alunos da Oficina, no primeiro trimestre de 2021. Analisamos os dados de uma perspectiva quantitativa-qualitativa. Isso permite, além de alcançar a descrição esperada, propor uma tipologia das performances, em relação a essa habilidade. Finalmente, expressamos perspectivas que este estudo abre

Palavras-chave: Habilidades matemáticas; novas tecnologias; software matemático; informações matemáticas; aprendizado matemático. 


\section{Introducción}

Es sabido que los estudiantes que inician estudios superiores presentan dificultades para la inserción en la institución. Los altos índices de reprobación en asignaturas iniciales suelen ser una de las causas -entre otras múltiples- de abandono, y las materias de matemática que forman parte de las primeras ofertas formativas no están exentas de esta situación. La Universidad Nacional de General Sarmiento (UNGS), en Argentina ha destinado -y lo sigue haciendo- denodados esfuerzos por cuidar las propuestas didácticas y los planteles docentes, realizando seguimientos e investigaciones que puedan ofrecer respuestas a esta problemática, o al menos generar las condiciones que se consideren más favorables, para un tiempo y situación de contexto dados. Puede verse un detalle importante de materiales, modos de admisión y perfiles del ingresante en instituciones de Argentina en Carnelli (2014).

En 2019, se implementa por primera vez en la UNGS el Programa de Acceso y Acompañamiento a Estudiantes de Grado y Pregrado, luego de un largo proceso de revisión curricular. Este Programa retoma la experiencia acumulada de investigaciones, estudios y seguimientos realizados en las materias iniciales ofrecidas anteriormente en la Universidad, y propone poner el foco en promover, en los estudiantes, el desarrollo de habilidades y hábitos que favorezcan un desempeño adecuado en la institución. De este modo, se intenta acompañar la inserción y permanencia en la UNGS de los estudiantes que comienzan sus estudios en ella.

Entre los espacios curriculares del Programa, se encuentra el Taller Inicial Obligatorio de Matemática (TIO-Matemática), contexto en el que se sitúa y desarrolla este trabajo. El Taller fue diseñado de modo de atender al desarrollo de ciertas habilidades matemáticas y hábitos de estudio trabajados con contenidos funcionales, que promueven la autonomía de los estudiantes y el reconocimiento de la utilidad del conocimiento que construyen.

En este marco, y con esto último como una pretensión explícita, planteamos como punto clave sobre el que pretendemos incidir: el acceso a información, obtenida 
mediante nuevas tecnologías, su comprensión y uso adecuado para aprender matemática.

Surge entonces, naturalmente, la necesidad de formar a los estudiantes en el buen uso de la tecnología para aprender matemática, con el amplio alcance que esto conlleva. Por una parte, es necesario trabajar el acceso a información, pero también la comprensión de lo hallado, selección y utilización adecuados en función de lo que le haya sido planteado al estudiante. Por otra parte, del trabajo con software específico para aprender matemática y la comunicación entre pares y con el docente, también resultan aspectos centrales a considerar desde el inicio.

La propuesta didáctica del TIO-Matemática ${ }^{1}$ atiende a estas cuestiones, como se verá más adelante.

Algunas de las preguntas iniciales que nos hemos hecho tienen como meta ampliar nuestro conocimiento sobre los estudiantes del TIO-Matemática. Mencionamos, por ejemplo: ante la necesidad de resolver actividades matemáticas, ¿deciden buscar información en la web?, ¿cómo lo hacen?, ¿cómo se organizan?, ¿qué tienen en cuenta?, ¿consideran que todo lo hallado es correcto?, ¿toman precauciones sobre la confiabilidad, en términos académicos, de los sitios visitados? ¿Deciden, autónomamente, utilizar algún software?, ¿para qué lo usan?, ¿conocen varios de ellos y sabrían cómo seleccionar alguno que consideren más adecuado, en función de lo que deben resolver? ¿Interactúan con pares o con el docente a través de algún recurso digital?, ¿reconocen que a través de las interacciones pueden aprender matemática?, etcétera.

En este artículo presentamos parte de un estudio que nos ha permitido describir, desde la perspectiva de los estudiantes, el acceso y uso de nuevas tecnologías en el contexto del Taller, con la finalidad de aprender matemática.

El trabajo se organiza del siguiente modo. Primero presentamos el problema de investigación, para lo que incluimos detalles del Taller en el que trabajamos, explicitamos puntos de partida y marco teórico. En este último, presentamos el posicionamiento

\footnotetext{
${ }^{1}$ El programa está disponible en: http://abcdnuevo.ungs.edu.ar/bases/progra/A0620A19S1.pdf
} 
asumido respecto de las habilidades matemáticas para considerar, como una de ellas, el acceso y uso de nuevas tecnologías para aprender matemática. Incluimos allí, el desarrollo propio de una rúbrica que permite evaluar el desempeño de los estudiantes en esta habilidad. A continuación, precisamos los objetivos y decisiones metodológicas asumidas. Como parte del desarrollo, presentamos el diseño de un cuestionario, que fundamentamos a partir de la rúbrica para, posteriormente, dar paso a una breve descripción de su implementación, análisis de datos y resultados. Finalmente, hacemos un cierre y presentamos las perspectivas que abren este trabajo.

\section{Planteo del problema}

\section{Descripción del contexto de trabajo}

En la Universidad Nacional de General Sarmiento (UNGS), Argentina, rige el Programa de Acceso y Acompañamiento a los Estudios Superiores, el cual se propone ofrecer espacios para fortalecer a los estudiantes en la vida académica. Los Talleres Iniciales Obligatorios son las primeras materias que cursan los estudiantes y donde se inicia el trabajo con este propósito.

En particular, como hemos mencionado, entre estos espacios se encuentra el Taller Inicial Obligatorio de Matemática que se enfoca en que los estudiantes reflexionen sobre su propio trabajo, tanto matemático, como del desarrollo de habilidades y hábitos. En este sentido, se plantea el trabajo con las siguientes habilidades: interpretación de bibliografía matemática, uso de lenguaje matemático (tanto natural como simbólico) y uso de nuevas tecnologías para aprender matemática. Estas habilidades se trabajan tomando contenidos sobre funciones lineales, cuadráticas y cuestiones generales sobre funciones. La duración es de 48 horas y se desarrolla en trimestres. Se ofrecen tres al año, los dos primeros entre febrero y julio y el tercero en la segunda mitad del año.

Hasta los primeros meses de 2020, el TIO-Matemática se cursaba en un encuentro semanal, presencial, de cuatro horas reloj. Bajo el contexto de la pandemia, a mediados de 2020, la cursada fue virtualizada, para dar continuidad pedagógica. La decisión de cómo virtualizar se tomó asumiendo los siguientes puntos de partida. 
- $\quad$ No es posible asegurar que la totalidad de los estudiantes dispongan de dispositivos digitales, conectividad y condiciones de sus entornos familiares que les permitan garantizar la participación en encuentros sincrónicos.

- Se prioriza no excluir estudiantes por estas razones, de tipo tecnológicas.

Por este motivo, todo el Taller fue diseñado nuevamente para desarrollarse en una modalidad íntegramente asincrónica. Se planteó como requerimiento de nuevas tecnologías para los estudiantes el mismo que se utiliza en clases presenciales. Esto es, la posibilidad de acceso a un aula virtual (tanto sea a través de una computadora, como de un celular); por lo tanto deben contar con conectividad, posibilidad de ver videos, enviar y recibir correos electrónicos y audios (no necesariamente grabar y enviar videos), y se habilitó el uso de aplicaciones matemáticas para celular (aunque no se lo exige, para no imprimir una condición adicional, restrictiva, que atañe a los dispositivos que utilizan).

Cabe resaltar que se preservó el trabajo de los estudiantes de manera individual y grupal, como también el foco en el desarrollo de las habilidades mencionadas.

En el primer trimestre de 2021, el taller contó con alrededor de 700 inscriptos, distribuidos en veintidós comisiones y los estudiantes son de carreras vinculadas con las Ciencias Exactas.

En este trabajo tomamos por objeto de estudio a la habilidad acceso y uso de nuevas tecnologías para aprender matemática desde la perspectiva de aquellos estudiantes que están cursando el TIO-Matemática durante el primer trimestre de 2021. Entendemos por acceso a nuevas tecnologías, a la decisión de los estudiantes de recurrir a ellas y, en consecuencia, dejamos fuera del alcance de este término, en este artículo, el disponer de esos dispositivos. (Consideramos exclusivamente los recursos con los que cuentan). Nos resulta relevante incluir el acceso a las nuevas tecnologías para esta investigación para tener información de los estudiantes que permita distinguir cómo se manejan para acceder a ellas de cómo lo hacen para utilizarlas ante un planteo matemático que deben abordar. 


\section{Puntos de partida y marco teórico}

Esta sección está organizada en dos partes. En la primera, abordamos el concepto de habilidades matemáticas, asumimos una conceptualización y explicitamos cuestiones que nos resultan centrales considerar. En la segunda parte, explicitamos cómo consideramos las nuevas tecnologías, cuáles de ellas forman parte del trabajo realizado y a partir de allí, describimos dimensiones y alcances de la habilidad matemática acceso y uso de nuevas tecnologías para aprender matemática.

\section{Sobre las habilidades matemáticas}

En la literatura vigente tanto de la Didáctica General como de Educación Matemática, se halla una amplia diversidad de usos y conceptualizaciones de los conceptos: habilidad, habilidad general y habilidad matemática. En algunos textos no se encuentran definiciones o caracterizaciones, por lo que se entiende que están considerados como términos primitivos. Por otra parte, en artículos que sí presentan definiciones, se han advertido algunas cuestiones que ameritan cuidados. Por ejemplo, algunas remiten a conceptos no definidos (como destrezas, capacidades o competencias); en otras queda utilizado el término como parte de la misma definición pretendida, generando un problema de circularidad; o la definición es demasiado abarcativa y no es claro qué quedaría fuera de su alcance. En Rodríguez (2016) pueden verse ejemplos de esto, sumado a la discusión respecto de las razones que originaron la definición que allí se expone, que asumimos en este trabajo como parte del marco teórico y que es la siguiente. Según Rodríguez (2016, p. 814, cursiva del autor), "una habilidad es un desempeño deliberado, no casual, adecuadamente realizado que permite resolver correctamente una cierta problemática planteada".

A partir de aquí, cuando la problemática involucra matemática, tenemos una habilidad matemática. En la referencia recién indicada se amplía la explicación, se suman detalles y se ofrecen ejemplos en el marco del Enfoque Cognitivo. (Puede ampliarse sobre este enfoque teórico de Educación Matemática en Pochulu y Rodríguez, 2015). 


\title{
Nuevas tecnologías. Acceso y uso para aprender matemática
}

Comenzamos mencionando que, para simplificar el lenguaje, consideramos las nuevas tecnologías como las tecnologías de la comunicación y la información (TIC) con el significado que propone Cobo Romaní (2009).

\begin{abstract}
Dispositivos tecnológicos (hardware y software) que permiten editar, producir, almacenar, intercambiar y transmitir datos entre diferentes sistemas de información que cuentan con protocolos comunes. Estas aplicaciones, que integran medios de informática, telecomunicaciones y redes, posibilitan tanto la comunicación y colaboración interpersonal (persona a persona) como la multidireccional (uno a muchos o muchos a muchos). Estas herramientas desempeñan un papel sustantivo en la generación, intercambio, difusión, gestión y acceso al conocimiento. (COBO ROMANÍ, 2009, p. 312)
\end{abstract}

Estos dispositivos tecnológicos, aplicaciones y herramientas que las constituyen son los que denominamos recursos tecnológicos, o simplemente recursos.

En Bravo (2016) se detalla un listado de recursos tecnológicos y posibles usos, ambos enfocados en el uso que el docente promueve de ellos para que los estudiantes aprendan matemática. Este énfasis nos permite considerar que podemos partir de su propuesta para describir y organizar los posibles usos que los estudiantes hacen de las TIC para aprender matemática. A continuación, se observa que no se requiere otra adaptación, más allá de la mención realizada.

Los recursos se presentan agrupados en dispositivos personales y aplicaciones para estos (calculadora, celular, procesadores de texto, graficadores, aplicaciones sobre geometría dinámica, etcétera); tecnologías anteriores (pizarrón, libros en papel, etcétera) y recursos vinculados a la web relacionados con la comunicación (blog, correo electrónico, aulas virtuales, etcétera) y con sitios con contenidos matemáticos (e-books, videos, tutoriales, artículos, etcétera).

En el mismo trabajo, se presenta el cuadro 1 que organiza, en cuatro dimensiones, una síntesis de posibles usos de los recursos tecnológicos para aprender matemática. 
Cuadro 1 - Uso de recursos para aprender Matemática

USO DE LOS RECURSOS (PARA APRENDER MATEMÁTICA)

\begin{tabular}{|c|c|c|}
\hline Vinculado a prod & & \multirow{16}{*}{$\begin{array}{l}\text { Trabajar colaborativamente } \\
\text { a distancia (en espacios } \\
\text { virtuales) } \\
\text { Comunicarse con otros } \\
\text { Compartir materiales } \\
\text { Hacer preguntas, compartir } \\
\text { respuestas o propuestas } \\
\text { Usar aulas virtuales }\end{array}$} \\
\hline Hacer operaciones numéricas, simbólicas & \multirow{15}{*}{ 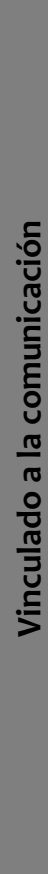 } & \\
\hline Graficar & & \\
\hline Visualizar & & \\
\hline Hacer construcciones geométricas & & \\
\hline Documentar (clases, resoluciones, etc.) & & \\
\hline Practicar y recibir retroalimentación & & \\
\hline Producir materiales & & \\
\hline Vinculado a recibir información matemática & & \\
\hline Buscar información & & \\
\hline Aprender contenidos & & \\
\hline Leer materiales & & \\
\hline $\begin{array}{c}\text { Vinculado a comunicar información } \\
\text { matemática }\end{array}$ & & \\
\hline Exponer trabajos con soporte & & \\
\hline $\begin{array}{l}\text { Producir escritos con nomenclatura } \\
\text { matemática }\end{array}$ & & \\
\hline Organizar la información & & \\
\hline
\end{tabular}

Fuente: BRAVO (2016, p. 16)

Al considerar en este trabajo no solo el uso de nuevas tecnologías para aprender matemática, sino también el acceso a ellas, hemos ampliado el marco teórico diseñando una rúbrica, que toma como punto de partida lo recién mencionado, y permite evaluar el desempeño en la habilidad acceso y uso de nuevas tecnologías para aprender matemática. Haremos una breve mención a la fundamentación y validación interjueces a la que la hemos sometido. Aunque en este trabajo no se aborda la evaluación del desempeño de los estudiantes en esta habilidad, la presentamos aquí porque la hemos utilizado para fundamentar el diseño del instrumento considerado, como se verá más adelante. 


\section{Acceso y uso de nuevas tecnologías para aprender matemática. Presentación de rúbrica}

Consideramos las dimensiones de los usos de recursos (BRAVO, 2016), presentadas anteriormente, reorganizadas en tres ejes, que contemplan accesos a nuevas tecnologías a las que un sujeto podría apelar para aprender matemática.

1. Acceso a información matemática.

2. Acceso a software matemático.

3. Acceso a la comunicación.

En cada uno de estos ejes, incluimos específicamente el uso dado para aprender matemática en un contexto de clases, donde el docente da una consigna o tarea para que los estudiantes realicen.

De este modo, en el primer eje, Información matemática, consideramos los siguientes aspectos:

- Palabras clave que utiliza para hacer las búsquedas.

- Confiabilidad de las fuentes.

- Selección de información adecuada.

- Uso de información para responder a una consigna/tarea.

El segundo eje, Software matemático, pone el foco en:

- Decisión de utilizar software en consignas que no lo indican expresamente.

- Elección del software a utilizar en función de lo que la consigna solicita.

- Uso del recurso para responder a la consigna.

El tercer eje, Comunicación, se centra en:

- Interacción con pares sea en tiempo real o asincrónico.

- Interacción con el docente sea en tiempo real o asincrónico.

- Uso de la comunicación para aprender matemática. 
La rúbrica toma cada uno de estos aspectos, conservando la división en ejes, y presenta indicadores para tres niveles de posibles desempeños. Como toda habilidad, no basta desempeñarse adecuadamente, sino que es necesario haber sido consciente de los desempeños que se realizarán, previo a ejecutarlos. En la rúbrica, esto está contemplado en las celdas griseadas.

La rúbrica fue sometida a la validación de pares, por dos expertos interjueces. En ambos casos, recibimos retroalimentaciones que nos permitieron lograr mayor precisión en la formulación. En el cuadro 2, se presenta la versión final.

\section{Cuadro 2 - Rúbrica}

\begin{tabular}{|c|c|c|c|}
\hline \multicolumn{4}{|c|}{ ACCESO Y USO DE NUEVAS TECNOLOGÍAS PARA APRENDER MATEMÁTICA } \\
\hline & $\begin{array}{c}\text { Desempeños de menor } \\
\text { nivel }\end{array}$ & $\begin{array}{c}\text { Desempeños de nivel } \\
\text { intermedio }\end{array}$ & $\begin{array}{c}\text { Desempeños de mayor } \\
\text { nivel }\end{array}$ \\
\hline \multicolumn{4}{|c|}{ INFORMACIÓN MATEMÁTICA } \\
\hline \multirow[t]{2}{*}{$\begin{array}{l}\text { Palabras } \\
\text { clave }\end{array}$} & $\begin{array}{c}\text { No utiliza palabras } \\
\text { claves } \\
\text { Copia textual la } \\
\text { consigna en el } \\
\text { buscador. (Espera } \\
\text { encontrar directamente } \\
\text { la respuesta) }\end{array}$ & $\begin{array}{l}\text { Sabe que debe utilizar } \\
\text { palabras clave } \\
\text { No selecciona palabras } \\
\text { clave pertinentes para la } \\
\text { búsqueda }\end{array}$ & $\begin{array}{l}\text { Utiliza palabras claves } \\
\text { representativas al tema } \\
\text { Reformula la búsqueda a } \\
\text { partir de las primeras } \\
\text { respuestas }\end{array}$ \\
\hline & \multicolumn{3}{|c|}{ En qué medida advierte que se usan palabras clave para las búsquedas } \\
\hline \multirow[t]{3}{*}{ Confiabilidad } & $\begin{array}{c}\text { No sabe que la } \\
\text { información hallada } \\
\text { podría ser errónea } \\
\text { No pone en duda la } \\
\text { información brindada } \\
\text { por el buscador }\end{array}$ & $\begin{array}{l}\text { Sabe que la información } \\
\text { hallada podría ser errónea } \\
\text { Sabe que existen fuentes } \\
\text { no confiables, pero no } \\
\text { tiene criterios para } \\
\text { distinguirlas }\end{array}$ & $\begin{array}{c}\text { Sabe que la información } \\
\text { hallada podría ser errónea } \\
\text { Tiene criterios para } \\
\text { resguardar la confiabilidad } \\
\text { Conoce sitios no confiables } \\
\text { y los evita }\end{array}$ \\
\hline & $\begin{array}{c}\text { Se queda con las } \\
\text { primeras "entradas" } \\
\text { que aparecen }\end{array}$ & $\begin{array}{l}\text { Sabe de algunas fuentes de } \\
\text { información confiables, y } \\
\text { es allí donde busca }\end{array}$ & $\begin{array}{c}\text { Contrasta la información } \\
\text { buscando en distintos sitios } \\
\text { Reconoce sitios académicos }\end{array}$ \\
\hline & \multicolumn{3}{|c|}{ En qué medida advierte que debe cuidar la confiabilidad del sitio } \\
\hline \multirow[t]{2}{*}{$\begin{array}{l}\text { Selección de } \\
\text { información } \\
\text { adecuada }\end{array}$} & $\begin{array}{l}\text { No selecciona } \\
\text { información }\end{array}$ & $\begin{array}{c}\text { Accede a la información, } \\
\text { pero no hace una selección } \\
\text { adecuada }\end{array}$ & $\begin{array}{c}\text { Selecciona información } \\
\text { adecuada, según lo que la } \\
\text { consigna solicita }\end{array}$ \\
\hline & \multicolumn{3}{|c|}{ En qué medida advierte que debe seleccionar información adecuada } \\
\hline Uso de & $\begin{array}{l}\text { No elabora la respuesta } \\
\text { que da, copia y pega lo }\end{array}$ & $\begin{array}{c}\text { Utiliza información } \\
\text { adecuada pero no logra }\end{array}$ & $\begin{array}{c}\text { Utiliza adecuadamente } \\
\text { información hallada para }\end{array}$ \\
\hline
\end{tabular}




\begin{tabular}{|c|c|c|c|}
\hline \multirow[t]{2}{*}{ información } & hallado & $\begin{array}{l}\text { organizarla para responder } \\
\text { a la consigna dada }\end{array}$ & responder a lo pedido \\
\hline & \multicolumn{3}{|c|}{$\begin{array}{c}\text { En qué medida advierte que debe trabajar con la información para elaborar una } \\
\text { respuesta a lo que se pide }\end{array}$} \\
\hline \multicolumn{4}{|c|}{ SOFTWARE MATEMÁTICO } \\
\hline \multirow[t]{2}{*}{$\begin{array}{l}\text { Decide } \\
\text { utilizar } \\
\text { software }\end{array}$} & $\begin{array}{l}\text { No quiere utilizar } \\
\text { software } \\
\text { No atina a usar } \\
\text { software }\end{array}$ & $\begin{array}{c}\text { Espera la indicación del } \\
\text { profesor para utilizar algún } \\
\text { software }\end{array}$ & $\begin{array}{l}\text { Decide autónomamente } \\
\text { cuándo utilizar software }\end{array}$ \\
\hline & \multicolumn{3}{|c|}{ En qué medida advierte que podría utilizar algún software } \\
\hline \multirow[t]{2}{*}{$\begin{array}{l}\text { Elección de } \\
\text { software }\end{array}$} & $\begin{array}{l}\text { Conoce un único } \\
\text { software y utiliza ese } \\
\text { Conoce al menos dos } \\
\text { software pero no tiene } \\
\text { criterios para decidir } \\
\text { cuál usar }\end{array}$ & $\begin{array}{l}\text { Conoce un único software } \\
\text { y dispone de criterios } \\
\text { insuficientes para } \\
\text { determinar si es o no } \\
\text { conveniente usarlo } \\
\text { Conoce distintos software, } \\
\text { pero usa siempre el mismo }\end{array}$ & $\begin{array}{c}\text { Elige qué software utilizar } \\
\text { en función de la consigna a } \\
\text { responder }\end{array}$ \\
\hline & \multicolumn{3}{|c|}{$\begin{array}{l}\text { En qué medida reconoce diferencias entre software y que algunos son más } \\
\text { convenientes que otros, según lo que deba resolver }\end{array}$} \\
\hline \multirow[t]{2}{*}{$\begin{array}{c}\text { Uso de } \\
\text { software } \\
\text { (ya elegido) }\end{array}$} & $\begin{array}{l}\text { No utiliza software } \\
\text { adecuadamente }\end{array}$ & $\begin{array}{c}\text { Utiliza software } \\
\text { adecuadamente } \\
\text { Utiliza el software para: } \\
\text {-verificar lo hecho a mano } \\
\text {-hacer cálculos confiables } \\
\text {-graficar prolijo, mejorar } \\
\text { presentaciones } \\
\text { Considera que lo que el } \\
\text { software ofrece como } \\
\text { respuesta siempre es } \\
\text { correcto, cree en “lo que } \\
\text { ve” }\end{array}$ & $\begin{array}{c}\text { Utiliza software } \\
\text { adecuadamente } \\
\text { Utiliza el software para lo } \\
\text { anterior, pero además: } \\
\text {-explorar una situación } \\
\text {-organizar y manipular } \\
\text { datos } \\
\text {-visualizar algún } \\
\text { comportamiento, } \\
\text { conjeturar, ... } \\
\text { Considera lo obtenido en el } \\
\text { software y lo analiza con } \\
\text { herramientas matemáticas }\end{array}$ \\
\hline & \multicolumn{3}{|c|}{$\begin{array}{l}\text { En qué medida conoce las distintas potencialidades del software utilizado y que no } \\
\text { siempre lo que se obtiene como respuesta es matemáticamente correcto }\end{array}$} \\
\hline \multicolumn{4}{|c|}{ COMUNICACIÓN } \\
\hline $\begin{array}{l}\text { Interacciones } \\
\text { entre pares }\end{array}$ & $\begin{array}{l}\text { No interactúa con sus } \\
\text { pares }\end{array}$ & $\begin{array}{l}\text { Interactúa con sus pares } \\
\text { usualmente } \\
\text { Interactúa con sus pares } \\
\text { solo cuando está obligado } \\
\text { por la propuesta de } \\
\text { enseñanza } \\
\text { No interactúa } \\
\text { adecuadamente (no saluda, } \\
\text { si hace una pregunta no es } \\
\text { claro a qué se refiere, no }\end{array}$ & $\begin{array}{l}\text { Interactúa con sus pares } \\
\text { usualmente y de forma } \\
\text { adecuada } \\
\text { Interactúa siempre con sus } \\
\text { pares adecuadamente }\end{array}$ \\
\hline
\end{tabular}




\begin{tabular}{|c|c|c|c|}
\hline & & pone nombre, etcétera) & \\
\hline & \multicolumn{3}{|c|}{ En qué medida interactúa digitalmente con sus pares } \\
\hline \multirow[t]{2}{*}{$\begin{array}{c}\text { Interacciones } \\
\text { con el } \\
\text { docente }\end{array}$} & $\begin{array}{l}\text { No interactúa con el } \\
\text { docente (ni por mail, } \\
\text { plataforma, mensajería, } \\
\text { etcétera) ni } \\
\text { espontáneamente ni } \\
\text { cuando se le indica }\end{array}$ & $\begin{array}{c}\text { No interactúa con el } \\
\text { docente espontáneamente } \\
\text { Interactúa con el docente } \\
\text { cuando se le indica } \\
\text { No interactúa } \\
\text { adecuadamente }\end{array}$ & $\begin{array}{l}\text { Interactúa con el docente } \\
\text { espontáneamente } \\
\text { Interactúa con el docente } \\
\text { cuando se le indica } \\
\text { Interactúa adecuadamente }\end{array}$ \\
\hline & \multicolumn{3}{|c|}{ En qué medida interactúa digitalmente con el docente } \\
\hline \multirow[t]{2}{*}{$\begin{array}{c}\text { Uso de la } \\
\text { comunicación } \\
\text { para } \\
\text { aprender } \\
\text { matemática }\end{array}$} & $\begin{array}{l}\text { Espera comunicarse con } \\
\text { el docente para recibir } \\
\text { explicaciones y } \\
\text { correcciones } \\
\text { Reniega de tener que } \\
\text { comunicarse con pares } \\
\text { para hacer trabajo en } \\
\text { grupo }\end{array}$ & $\begin{array}{l}\text { Acepta comunicarse con } \\
\text { sus pares para resolver } \\
\text { consignas cuando está } \\
\text { pautado así }\end{array}$ & $\begin{array}{c}\text { Utiliza distintos medios de } \\
\text { comunicación con pares y } \\
\text { docentes para aprender } \\
\text { matemática } \\
\text { Busca comunicarse con } \\
\text { pares para estudiar } \\
\text { matemática, incluso cuando } \\
\text { no está pautado }\end{array}$ \\
\hline & \multicolumn{3}{|c|}{$\begin{array}{l}\text { En qué medida advierte que aprende matemática a través de distintas interacciones } \\
\text { con el docente y/o con sus pares }\end{array}$} \\
\hline
\end{tabular}

Fuente: Elaboración propia, 2021.

Con la finalidad de ilustrar cuestiones referidas al desempeño de estudiantes en la habilidad abordada, ofrecemos a continuación una consigna matemática del Taller y respuestas de un estudiante que muestran la decisión de recurrir a nuevas tecnologías y su uso para responderla.

Las respuestas que compartimos se refieren a los ítems 4) y 5) de la siguiente consigna (Figura 1).

Figura 1 - Consigna

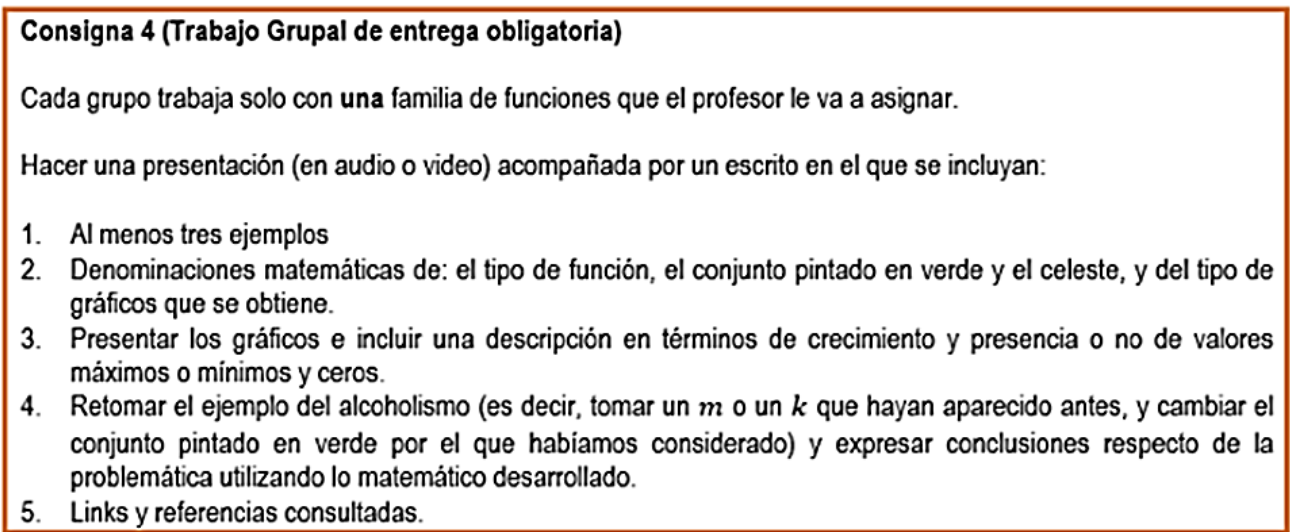

Fuente: Material TIO-Matemática, 2021. 
La familia de funciones a la que hace referencia fue asignada por el docente y resultó una de las dos siguientes. En el Taller habían aparecido previamente casos particulares de ellas y con dominios restringidos.

Estas son: $f: \mathbb{R} \rightarrow \mathbb{R}, f(x)=m . x$ y $g: \mathbb{R}-\{0\} \rightarrow \mathbb{R}, g(x)=\frac{k}{x} \operatorname{con} m, k \in \mathbb{R}$.

Una respuesta de un equipo es la siguiente (Figura 2).

Figura 2 - Respuesta a la consigna

4) Teniendo en cuenta el dominio considerado para el ejercicio del alcoholismo $y$ reemplazando la constante $\mathrm{k}$ obtenemos la siguiente función:

$g:[40,200] \Rightarrow \mathbb{R}$

$g(x)=\frac{48}{x}$

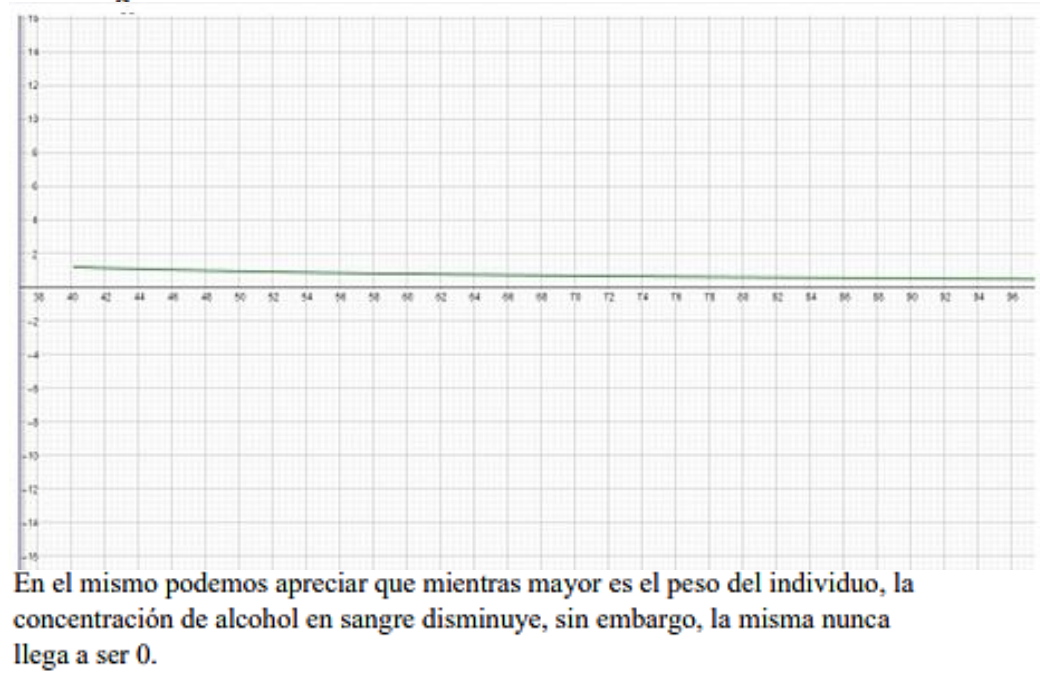

5) https://www.youtube.com/watch?v=Zxz4tta4ZDA

Fuente: Respuestas de un grupo de estudiantes, 2021.

La imagen presentada es la respuesta recibida. Respecto del ítem 4, se observa: la decisión de apelar a GeoGebra para graficar y un manejo adecuado para que el gráfico presente la función con el dominio restringido. Por otra parte, la afirmación de que la curva nunca tomará el valor o queda argumentado por lo que muestra el software, sustento que es erróneo, aunque en este caso sea cierta la afirmación.

Respecto del ítem 5, se incluye como referencia un video que se ofreció en el marco mismo del Taller, con lo que no pusieron en juego otras herramientas de 
búsqueda, pero garantizaron la confiabilidad del sitio. Esta cuestión es reconocida como un aprendizaje por uno de los integrantes del equipo, a propósito de una instancia de reflexión y autoevaluación que fue solicitada promediando el Taller. Incluimos, en figura 3, un recorte de su respuesta a una consigna de autoevaluación.

Figura 3 - Consigna y respuesta de un estudiante

3" Mencioná tres cosas que aprendíste durante la primera parte de este Taller.

Durante la primera parte de este taller aprendí a trabajar en equipo, a realizar una correcta toma de apuntes y como buscar información en fuentes confiables.

Fuente: Material TIO-Matemática, 2021.

Los recursos tecnológicos a los que nos referimos de aquí en adelante son los descriptos en el contexto y esto se debe a las razones explicitadas.

Con este marco teórico y puntos de partida establecidos presentamos, en la siguiente sección, los objetivos del estudio y las decisiones metodológicas asumidas.

\section{Objetivos y metodología}

A partir del interés mencionado y para el contexto descripto, el objetivo planteado es describir el acceso y uso de nuevas tecnologías para aprender matemática, desde la perspectiva de estudiantes del TIO-Matemática.

Metodológicamente, hemos tomado las siguientes decisiones. Inicialmente diseñamos y fundamentamos un cuestionario que permite recabar datos sobre la perspectiva de los estudiantes respecto de la habilidad estudiada.

El cuestionario fue implementado en Google Forms, aplicado a la totalidad de estudiantes activos del TIO-Matemática hacia finales del primer trimestre de 2021, y ha sido de respuesta voluntaria. Enviamos a los estudiantes el vínculo del cuestionario, junto 
con el pedido de respuesta, por mensajería interna del aula y por foro, y se estableció un tiempo de una semana para recibir las respuestas.

Planteamos el análisis de los datos del siguiente modo. Revisamos las respuestas individuales para cotejar consistencia interna. Es decir, buscamos ver si las respuestas dadas a distintas formulaciones no son contradictorias entre sí. Presentamos un análisis cuanti-cualitativo que nos permite tener una mirada global de los estudiantes. Tuvimos en cuenta la posibilidad de proponer tipologías de desempeños (declarados por los estudiantes) similares, respecto de la habilidad estudiada. Para esto último, consideramos nuevamente las respuestas individuales.

\section{Desarrollo}

Presentamos en esta sección primeramente el diseño y fundamentación del cuestionario, luego detallamos el proceso de implementación, los análisis realizados y resultados.

\section{Diseño y fundamentación del cuestionario}

El instrumento es un cuestionario cerrado tipo Likert y se presenta dividido en tres secciones. Cada una de ellas se asocia con cada eje de la rúbrica. La primera, se relaciona con el eje Información matemática, presenta doce afirmaciones acerca de cómo el estudiante busca y usa información de la web. La segunda sección, se vincula con el eje Software matemático y consta de diez frases sobre el acceso y uso de software matemáticos; y la tercera, se relaciona con la Comunicación, tiene once afirmaciones respecto al acceso y uso de los medios digitales para comunicarse. Lo que permite fundamentar que el diseño del cuestionario es la correspondencia entre las afirmaciones y los indicadores de la rúbrica. Para mostrar aquí dicha correspondencia, agregamos una fila, luego de cada aspecto, en la que presentamos, numeradas, las afirmaciones del cuestionario. Incluimos números en las celdas de la rúbrica que se corresponden con el 
número de afirmación del cuestionario. A continuación en el cuadro 3 mostramos esto, a modo de ejemplo para la sección Información matemática.

El cuestionario completo puede en

https://drive.google.com/file/d/19eFqcgzKUoTlvOOLFXfpGAilrgw6Czu_/view?usp=sharing.

Cuadro 3 - Ejemplo del modo de fundamentar el cuestionario a partir de la rúbrica

\begin{tabular}{|c|c|c|c|}
\hline \multicolumn{4}{|c|}{ INFORMACIÓN MATEMÁTICA } \\
\hline \multirow[t]{2}{*}{ Palabras clave } & $\begin{array}{l}\text { No utiliza palabras claves } \\
\text { 3. Copia textual la } \\
\text { consigna en el buscador. } \\
\text { (Espera encontrar } \\
\text { directamente la respuesta) }\end{array}$ & $\begin{array}{l}\text { 2. No selecciona palabras clave } \\
\text { pertinentes para la búsqueda }\end{array}$ & $\begin{array}{l}\text { 2. Utiliza palabras claves } \\
\text { representativas al tema } \\
\text { 4. Reformula la búsqueda a } \\
\text { partir de las primeras } \\
\text { respuestas }\end{array}$ \\
\hline & \multicolumn{3}{|c|}{ En qué medida advierte que se usan palabras clave para las búsquedas } \\
\hline $\begin{array}{l}\text { Afirmaciones } \\
\text { del } \\
\text { cuestionario }\end{array}$ & \multicolumn{3}{|c|}{$\begin{array}{l}\text { Cuando busco información en la web para responder consignas: } \\
\text { 1. debo usar palabras clave } \\
\text { 2. uso palabras clave pertinentes, representativas del tema o pregunta } \\
\text { 3. copio textual la consigna/pregunta en el buscador } \\
\text { 4. reformulo la búsqueda cambiando las palabras clave, si entiendo que lo hallado no me } \\
\text { resulta útil }\end{array}$} \\
\hline \multirow[t]{2}{*}{ Confiabilidad } & $\begin{array}{l}\text { 5. No sabe que la } \\
\text { información hallada podría } \\
\text { ser errónea } \\
\text { 5. No pone en duda la } \\
\text { información brindada por } \\
\text { el buscador } \\
\text { 8. Se queda con las } \\
\text { primeras "entradas" que } \\
\text { aparecen }\end{array}$ & $\begin{array}{l}\text { 5. Sabe que la información } \\
\text { hallada podría ser errónea } \\
\text { 9. Sabe que existen fuentes no } \\
\text { confiables, pero no tiene } \\
\text { criterios para distinguirlas } \\
\text { 6. Sabe de algunas fuentes de } \\
\text { información confiables, y es } \\
\text { allí donde busca }\end{array}$ & $\begin{array}{l}\text { 5. Sabe que la información } \\
\text { hallada podría ser errónea } \\
\text { 9. Tiene criterios para } \\
\text { resguardar la confiabilidad } \\
\text { 7. Conoce sitios no confiables y } \\
\text { los evita } \\
\text { 6. Contrasta la información } \\
\text { buscando en distintos sitios } \\
\text { 9. Reconoce sitios académicos }\end{array}$ \\
\hline & \multicolumn{3}{|c|}{ En qué medida advierte que debe cuidar la confiabilidad del sitio } \\
\hline $\begin{array}{l}\text { Afirmaciones } \\
\text { del } \\
\text { cuestionario }\end{array}$ & \multicolumn{3}{|c|}{$\begin{array}{l}\text { Cuando busco información en la web para responder consignas: } \\
\text { 5. sé que la información que encuentre podría ser errónea } \\
\text { 6. conozco sitios de internet confiables y recurro a ellos } \\
\text { 7. conozco sitios que no son confiables y los evito } \\
\text { 8. me quedo con los primeros resultados de la búsqueda } \\
\text { 9. sé que hay sitios no confiables, pero no sé cómo distinguirlos }\end{array}$} \\
\hline \multirow{2}{*}{$\begin{array}{l}\text { Selección de } \\
\text { información } \\
\text { adecuada }\end{array}$} & No selecciona información & $\begin{array}{c}\text { 10. Accede a información, pero } \\
\text { no hace una selección } \\
\text { adecuada }\end{array}$ & $\begin{array}{c}\text { Selecciona información } \\
\text { adecuada, según lo que la } \\
\text { consigna solicita }\end{array}$ \\
\hline & \multicolumn{3}{|c|}{ En qué medida advierte que debe seleccionar información adecuada } \\
\hline \multirow[t]{2}{*}{$\begin{array}{c}\text { Uso de } \\
\text { información }\end{array}$} & $\begin{array}{l}\text { 11. No elabora la respuesta } \\
\text { que da, copia y pega lo } \\
\text { hallado }\end{array}$ & $\begin{array}{c}\text { 12. Utiliza información } \\
\text { adecuada pero no logra } \\
\text { organizarla para responder a } \\
\text { la consigna dada }\end{array}$ & $\begin{array}{l}\text { 12. Utiliza adecuadamente } \\
\text { información hallada para } \\
\text { responder a lo pedido }\end{array}$ \\
\hline & \multicolumn{3}{|c|}{$\begin{array}{l}\text { En qué medida advierte que debe trabajar con la información para elaborar una respuesta a lo } \\
\text { que se pide }\end{array}$} \\
\hline $\begin{array}{l}\text { Afirmaciones } \\
\text { del }\end{array}$ & $\begin{array}{l}\text { Cuando busco información } € \\
\text { 10. tomo lo que encontré y s } \\
\text { 11. tomo lo que encontré y lc }\end{array}$ & $\begin{array}{l}\text { n la web para responder consigna } \\
\text { empre hago una selección, elijo q } \\
\text { copio textual }\end{array}$ & Ié usar \\
\hline
\end{tabular}




\section{\begin{tabular}{l|l} 
cuestionario & 12. tomo lo que encontré y pongo atención de modo de usarla para responder lo que me piden
\end{tabular}}

Fuente: Elaboración propia, 2021.

De este modo hemos fundamentado el diseño del cuestionario.

Hacia finales del primer trimestre de 2021, se encontraban activos 550 estudiantes, de los cuales 75 de ellos respondieron al cuestionario en el plazo dado.

\section{Análisis de datos y resultados}

Como hemos mencionado, realizamos un análisis cuanti-cualitativo de las respuestas. Presentamos a continuación algunas consideraciones respecto del mismo.

Hemos considerado las respuestas de cada uno de los estudiantes a lo largo de los tres ejes poniendo atención a la consistencia en las respuestas, como anticipamos. Solo cuatro casos manifestaron inconsistencias. Mencionan, por ejemplo que deben usar y usan palabras clave y en otra respuesta expresan que copian textual la consigna en el buscador. O también que conocen un único software, y en contraposición declaran saber cómo elegir qué software utilizar ante una consigna.

Para el análisis cuanti-cualitativo reagrupamos las respuestas de la siguiente manera: "Totalmente de acuerdo" y "De acuerdo" como "Acuerdo total o parcial"; y "En desacuerdo y totalmente en desacuerdo" como "Desacuerdo total o parcial". Presentamos el análisis de las respuestas conservando la separación según los ejes: información matemática, software matemático, y comunicación.

Luego de ello, retomamos las respuestas individuales y advertimos rasgos comunes a lo que los estudiantes manifiestan sobre su modo de desenvolverse ante el acceso y uso de nuevas tecnologías para aprender matemática. Esto nos ha permitido proponer una tipología de desempeños vinculados a la habilidad estudiada, desde la visión personal de los estudiantes. Presentamos una caracterización de cada uno de esos tipos. 


\section{Análisis cuanti-cualitativo}

Respecto al primer eje, Información matemática, más del 70\% de los estudiantes manifiestan acuerdo parcial o total en relación a las siguientes afirmaciones, ordenadas según la frecuencia, de mayor a menor. Consideramos este criterio de ordenamiento, en lo sucesivo.

Cuando busco información en la web para responder consignas:

$\checkmark$ uso palabras clave pertinentes, representativas del tema o pregunta

$\checkmark$ debo usar palabras clave

$\checkmark$ tomo lo que encontré y pongo atención de modo de usarla para responder lo que me piden

$\checkmark$ reformulo la búsqueda cambiando las palabras clave, si entiendo que lo hallado no me resulta útil

$\checkmark$ tomo lo que encontré y siempre hago una selección, elijo qué usar

$\checkmark$ sé que la información que encuentre podría ser errónea

$\checkmark$ conozco sitios de internet confiables y recurro a ellos

$\checkmark$ conozco sitios que no son confiables y los evito

Esto está en concordancia con que más del $66 \%$ manifiesta estar en desacuerdo parcial o total respecto de las siguientes afirmaciones.

Cuando busco información en la web para responder consignas:

$\checkmark$ tomo lo que encontré y lo copio textual

$\checkmark$ me quedo con los primeros resultados de la búsqueda

A partir de los resultados expuestos, notamos que la mayoría de estudiantes no confía en la validez de la información a la que accede a través de la web. Por el contrario, perciben que la actividad de búsqueda, selección y uso de información es una tarea 
compleja. Consideramos que esto es un buen punto de partida para que adquieran y/o desarrollen algunas estrategias para mejorar su desempeño.

Destacamos que para los siguientes aspectos, no hay una mayoría marcada en el grado de acuerdo o desacuerdo.

Cuando busco información en la web para responder consignas:

$\checkmark$ copio textual la consigna/pregunta en el buscador

$\checkmark$ sé que hay sitios no confiables, pero no sé cómo distinguirlos

En relación a la primera de estas afirmaciones nos resulta llamativo que 10 de los estudiantes estén de acuerdo con copiar textual la consigna en el buscador y que 27 lo duden. Podría ocurrir que les resulte un método tan habitual que, al no detenerse a pensarlo, hayan elegido la respuesta "ni de acuerdo ni en desacuerdo". La segunda afirmación que está ligada a la confiabilidad de los sitios web consultados presenta la siguiente distribución: 22 estudiantes en acuerdo parcial o total; 20 que no están ni de acuerdo ni en desacuerdo y 33 estudiantes en un desacuerdo parcial o total. Interpretamos, a partir de esta dispersión, que es un asunto que aún no les ha resultado problemático en el desarrollo del Taller. Probablemente no tuvieron experiencias en las que el uso de datos erróneos o falsificados, provenientes de sitios no confiables, les haya sido perjudicial para su aprendizaje. Por otra parte, quienes están de acuerdo podrían no haber vivido este tipo de experiencia negativa y responder influenciados por el trabajo que se desarrolla en el TIO-Matemática al respecto.

Respecto al segundo eje, Software matemático, más del 60\% de los estudiantes están en parcial o total acuerdo con las siguientes afirmaciones.

En relación con el uso de software matemático para resolver una consigna:

$\checkmark$ considero lo que el software produce/ofrece pero no es suficiente para tener certeza, siempre debo usar argumentos matemáticos que no dependan de la tecnología

$\checkmark$ uso el software para explorar una situación o visualizar algún comportamiento 
uso el software únicamente para: verificar, hacer cuentas y graficar más prolijo

En cuanto a la decisión de utilizar software, más del 50\% de estudiantes están en total o parcial acuerdo con "espero que mi docente me indique que tengo que usar algún software"; y otro más del 50\% manifiesta estar en descuerdo total o parcial con la afirmación "No quiero usar software o no se me ocurre hacerlo". Interpretamos que dentro del porcentaje restante de la primera afirmación, un pequeño grupo de estudiantes hace uso de software independientemente de las indicaciones del docente. Mientras que dentro del porcentaje de la segunda afirmación aun si quieren o se les ocurre usarlo, esperan a que el docente se los indique.

Destacamos que, para los siguientes aspectos, no hay una mayoría marcada en una posición.

En relación con el uso de software matemático para resolver una consigna:

$\checkmark$ decido por mi cuenta cuándo usar software

$\checkmark$ conozco un único software y siempre uso ese

$\checkmark$ conozco varios software, pero uso siempre el mismo

$\checkmark$ tengo claro cómo elegir qué software me conviene usar para resolver la consigna que me dan

$\checkmark$ el software siempre produce/ofrece respuestas/resultados correctos

En relación a estos últimos aspectos, 34 alumnos están en parcial o total acuerdo con "Tengo claro cómo elegir qué software me conviene usar para resolver la consigna que dan". Sin embargo, encontramos que con la afirmación "Conozco varios software, pero siempre uso el mismo", 23 son los estudiantes que están de acuerdo y 22 que no lo están. Además, 15 responden estar de acuerdo con "Conozco un único software y siempre uso ese" y 30 no estarlo. Considerando que son estudiantes que se encuentran cursando la primera materia matemática de las diferentes carreras, interpretamos que las respuestas se deban a que no están familiarizados con todas las funciones que puede ofrecer un mismo software. 
Por otra parte, 20 estudiantes están de acuerdo con "El software siempre ofrece resultados correctos", otros 15 están en desacuerdo y 40 no supieron responder. Como docentes, estos datos nos resultan significativos ya que nos dan un indicio sobre lo que se podría trabajar en el Taller, por ejemplo prestando atención a los software que incluyen el paso a paso de los cálculos o que muestran gráficos con sus correspondientes tablas de valores, puesto que no solo podrían considerarlos como suficientes sino que perderían (o no) la importancia de explorar, analizar y comprender acerca de algún tema, contenido en particular.

Respecto al tercer eje, Comunicación, más del 50\% de estudiantes está en parcial o total acuerdo con las siguientes afirmaciones, ordenadas según la frecuencia.

En relación con el acceso a, y uso, de medios digitales para comunicarme con otros y para aprender matemática:

$\checkmark$ soy cuidadoso para interactuar con mis compañeros (me dirijo correctamente, saludo, etcétera)

$\checkmark$ considero siempre interactuar con mi docente adecuadamente (saludar, escribir correctamente, etcétera)

$\checkmark$ acepto trabajar con mis compañeros, si está pautado y puedo aprender matemática

$\checkmark$ me comunico por distintas vías con mis compañeros y con mi docente para aprender matemática

$\checkmark$ interactúo usualmente con mis compañeros

Destacamos que, para los siguientes aspectos, no hay una mayoría marcada en una posición, con muy poca diferencia entre Acuerdo total o parcial y Desacuerdo total o parcial.

$\checkmark$ Interactúo con mis compañeros únicamente cuando mi docente me lo indica

$\checkmark$ Interactúo con mi docente únicamente cuando me lo indica/solicita

$\checkmark$ Interactúo con mi docente usual y espontáneamente 
En las dos afirmaciones siguientes, el acuerdo (sea total o parcial) tuvo una marcada diferencia respecto de los desacuerdos, duplicándolo en porcentajes.

$\checkmark \quad$ La única manera de aprender matemática es si mi docente me explica y corrige

$\checkmark$ Intento estudiar con mis compañeros (independientemente si está pautado, o no)

Los modelos tradicionales de enseñanza, mayoritariamente predominantes en la escolaridad media, fomentan esa imagen del aprendizaje, asociada a recibir explicaciones en primer lugar para luego resolver actividades que son corregidas por el docente. Aunque el Taller no tiene este enfoque, no es sorprendente que esta imagen sea imperante. Por un lado, debido a las experiencias acumuladas de los años de escolaridad anterior y por otro, por la corta vivencia de una propuesta diferente. Cabe resaltar que al momento de la aplicación del cuestionario, los estudiantes aún no habían concluido el Taller.

\section{Sobre la tipología de desempeños referidos al acceso y uso de nuevas tecnologías, desde la perspectiva de los estudiantes}

En este apartado proponemos una caracterización de seis tipos de desempeños que identificamos a partir de las respuestas de los estudiantes.

Tipo 1:

Respecto al eje de Información matemática, encontramos que las palabras clave son utilizadas como criterio para las búsquedas y, como esperamos, no copiar textual la consigna en el buscador. Asimismo, se suma el conocer algunos sitios web confiables y tener alguna noción de cómo distinguirlos de aquellos que no lo son. Luego de realizar la búsqueda de información, se considera la selección de información y atención en responder lo pedido.

Respecto al eje de Software matemático, incluimos aquí la decisión autónoma de cuándo usarlo, pudiendo desconocerse la existencia de variedad de ellos así como de tener criterio para elegir cuál utilizar, dependiendo de la consigna dada. Sumamos la posibilidad de no tener claridad respecto de si lo que el software devuelve es o no 
matemáticamente correcto, así como tampoco tener en claro si lo que brinda es suficiente como argumento o se requiere algún otro tipo de trabajo matemático.

Respecto del eje Comunicación, la interacción con pares es considerada como algo usual y con el docente cuando es indicado.

Tipo 2:

Respecto del eje Información matemática, coincide con el Tipo 1. De igual modo respecto del Software matemático, aunque incluimos el reconocimiento de que no siempre los resultados que ofrece el software deben ser matemáticamente correctos por lo que se requiere el uso de argumentos matemáticos.

En tanto al eje de Comunicación, las interacciones entre estudiantes se dan cuando el docente lo indica, dando lugar al descontento del trabajo en grupos y a evitar estudiar con compañeros. En cambio, es fluida la comunicación del estudiante con el docente.

Tipo 3:

Respecto al eje Información matemática, en este Tipo se incluyen desempeños similares a los de los grupos anteriores. Sin embargo damos lugar a que no esté claro aún si copiar textualmente la consigna recibida en el buscador es una estrategia que les resultará valiosa, o no.

En relación con el eje de Software matemático, el Tipo 3 incluye desempeños pasivos respecto del uso de software y dependientes del docente. Se espera la indicación $y$, aunque conozcan varios, se utiliza siempre el mismo. Se considera usar argumentos matemáticos que no dependan de la tecnología. Entre los desempeños llamativos incluidos, resaltamos la creencia de que el software siempre ofrece resultados correctos y que el uso se restrinja a facilitar cálculos y ahorrar tiempo y pulcritud en las respuestas.

Respecto al eje de Comunicación, se manifiestan interacciones con el docente y entre pares de forma adecuada. Se explicita el acuerdo en que la única manera de aprender es con la explicación del docente. 
Tipo 4:

Respecto al eje Información matemática, lo que diferencia principalmente a este Tipo de los otros, es el saber que hay sitios no confiables pero no saber distinguirlos. Se manifiesta desconocimiento en cómo hacer búsquedas, saber que deben usar palabras clave, sin más precisiones, lo que también lleva a encontrar copia textual de la consigna en el buscador. Luego de realizar la búsqueda de información, se considera la selección de información y atención en responder lo pedido.

En relación con el acceso y uso de Software matemático, se encuentra un posicionamiento pasivo, a la espera de indicaciones para su uso. Esto no inhibe el conocer qué software elegir dependiendo la consigna. En el trabajo matemático con el uso de software se desconoce que éste puede ofrecerle resultados incorrectos, que posee limitaciones, y que en la formulación de sus respuestas se deberán incluir argumentos matemáticos.

En lo que respecta al eje de Comunicación, se manifiesta la interacción entre estudiantes, de manera usual y con el docente cuando es señalado. Se explicita el acuerdo en que la única manera de aprender es con la explicación del docente.

Tipo 5:

Respecto al eje Información matemática y al de Comunicación, se manifiestan desempeños contradictorios, como usar palabras clave, y copiar textualmente la consigna en el buscador. Aunque se encuentren interacciones usualmente con compañeros y docente, pueden no ser adecuadas con este último.

Respecto al eje de Software matemático, se utiliza uno único, desconociendo si los resultados que ofrece son siempre correctos desde el punto de vista matemático, o no. Del mismo modo, se desconoce si es necesario usar argumentos matemáticos que no dependan de la tecnología. Incluye desempeños pasivos respecto del uso de software y dependientes del docente. Sin embargo, se manifiesta la posibilidad de elección de software apropiados para resolver una consigna, y un uso del mismo no solo para hacer cuentas, graficar sino también explorar. 
Tipo 6:

Respecto al primer eje, Información matemática, se manifiestan criterios de búsqueda. Sin embargo, no hay cuidado en su uso ni en la atención a responder a lo pedido.

Respecto al Software matemático, se presentan desempeños que requieren indicaciones externas, sin considerar que debe analizarse la validez matemática de las respuestas halladas y la necesidad de dar argumentos matemáticos que no dependan de la tecnología, al responder a una consigna. Además no se dispone de criterios para seleccionar software y no se hace un uso adecuado.

Respecto del eje Comunicación, se manifiesta dependencia del docente, tanto para aprender como para interactuar, lo que es resuelto adecuadamente. El trabajo en grupo no está promovido autónomamente ni hay un rechazo explícito. Se señala como notorio que las interacciones entre compañeros pueden no ser adecuadas, según los parámetros esperados en la universidad.

El cuadro 4, sintetiza esquemáticamente los rasgos principales de la tipología, distinguiendo matices propios por eje y exhibiendo una síntesis de cada tipo.

Cuadro 4 - Rasgos principales sobre la tipología de desempeños

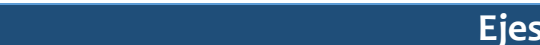

Información Software

matemática

matemático

\begin{tabular}{|c|c|c|c|c|}
\hline Tipo 1 & $\begin{array}{l}\text { Busca apropiadamente } \\
\text { información } \\
\\
\text { Selecciona y usa } \\
\text { adecuadamente } \\
\text { información }\end{array}$ & $\begin{array}{l}\text { Apela autónomamente a } \\
\text { un software } \\
\text { Desconoce variedad de } \\
\text { software } \\
\text { No reconoce alcances del } \\
\text { uso de software }\end{array}$ & $\begin{array}{l}\text { Interactúa con sus pares } \\
\text { Responde a las } \\
\text { indicaciones del docente } \\
\text { para interactuar con él }\end{array}$ & $\begin{array}{l}\text { Dispone de pautas de } \\
\text { trabajo para el acceso y uso } \\
\text { de información } \\
\text { Desconoce diversidad de } \\
\text { software y su alcance ante } \\
\text { el trabajo matemático } \\
\text { Presenta una actitud pasiva } \\
\text { en la interacción con el } \\
\text { docente, y prefiere el } \\
\text { trabajo grupal }\end{array}$ \\
\hline Tipo 2 & $\begin{array}{l}\text { Pone atención a } \\
\text { responder lo pedido }\end{array}$ & $\begin{array}{l}\text { Apela autónomamente a } \\
\text { un software } \\
\text { Desconoce variedad de } \\
\text { software } \\
\text { Reconoce alcances del }\end{array}$ & $\begin{array}{l}\text { Prefiere el trabajo } \\
\text { individual } \\
\text { Interactúa usualmente } \\
\text { con el docente }\end{array}$ & $\begin{array}{l}\text { Dispone de pautas de } \\
\text { trabajo para el acceso y uso } \\
\text { de información } \\
\text { Desconoce diversidad de } \\
\text { software pero reconoce } \\
\text { alcances y sabe que debe }\end{array}$ \\
\hline
\end{tabular}




\begin{tabular}{|c|c|c|c|c|}
\hline & & $\begin{array}{l}\text { uso de software } \\
\text { Tiene presente que debe } \\
\text { dar argumentos } \\
\text { matemáticos }\end{array}$ & & $\begin{array}{c}\text { tener argumentos } \\
\text { matemáticos que no } \\
\text { dependan de la tecnología } \\
\text { Prefiere el trabajo } \\
\text { individual y con el docente }\end{array}$ \\
\hline Tipo 3 & $\begin{array}{l}\text { Busca información de } \\
\text { manera adecuada e } \\
\text { inadecuada } \\
\text { Selecciona información } \\
\text { Pone atención a } \\
\text { responder lo pedido }\end{array}$ & $\begin{array}{l}\text { Utiliza software cuando } \\
\text { se lo indica el docente } \\
\text { Utiliza solo un software } \\
\text { Desconoce limitaciones } \\
\text { del software } \\
\text { Tiene presente usar } \\
\text { argumentos matemáticos } \\
\text { que no dependan de la } \\
\text { tecnología }\end{array}$ & $\begin{array}{l}\text { Interactúa con el docente } \\
\text { y estudiantes } \\
\text { La única forma de } \\
\text { aprender matemática que } \\
\text { reconoce es a través de la } \\
\text { explicación del docente }\end{array}$ & $\begin{array}{l}\text { Dispone de pautas de } \\
\text { trabajo para el acceso y uso } \\
\text { adecuado de información } \\
\text { Depende de las } \\
\text { indicaciones del docente } \\
\text { para utilizar software e } \\
\text { interactuar } \\
\text { Concibe como una única } \\
\text { forma de aprender } \\
\text { matemática la explicación } \\
\text { del docente }\end{array}$ \\
\hline Tipo 4 & $\begin{array}{c}\text { No busca } \\
\text { apropiadamente } \\
\text { Selecciona información } \\
\text { Pone atención a } \\
\text { responder lo pedido }\end{array}$ & $\begin{array}{l}\text { Espera indicación del } \\
\text { docente para usar } \\
\text { software } \\
\text { Sabe elegir software } \\
\text { apropiado } \\
\text { No sabe que el software } \\
\text { puede ofrecer resultados } \\
\text { incorrectos } \\
\text { Desconoce limitaciones } \\
\text { del software } \\
\text { No considera que debe } \\
\text { dar otros argumentos } \\
\text { matemáticos }\end{array}$ & $\begin{array}{l}\text { Interactúa con } \\
\text { compañeros usualmente } \\
\text { y con el docente cuando } \\
\text { se lo indican } \\
\text { La única forma de } \\
\text { aprender matemática que } \\
\text { reconoce es a través de la } \\
\text { explicación del docente }\end{array}$ & $\begin{array}{c}\text { No dispone de pautas para } \\
\text { acceso y uso adecuado de } \\
\text { información ni para utilizar } \\
\text { adecuadamente el } \\
\text { software } \\
\text { Depende de las } \\
\text { indicaciones del docente } \\
\text { para el uso de software } \\
\text { Concibe como una única } \\
\text { forma de aprender } \\
\text { matemática la explicación } \\
\text { del docente }\end{array}$ \\
\hline Tipo 5 & $\begin{array}{l}\text { No busca } \\
\text { apropiadamente } \\
\text { No selecciona } \\
\text { información } \\
\text { No pone atención a } \\
\text { responder lo pedido }\end{array}$ & $\begin{array}{c}\text { Espera indicación del } \\
\text { docente para usar } \\
\text { software } \\
\text { Conoce varios software } \\
\text { pero usa siempre el } \\
\text { mismo } \\
\text { Sabe elegir software } \\
\text { apropiado } \\
\text { Usa el software no solo } \\
\text { para hacer cuentas, } \\
\text { graficar sino también } \\
\text { explorar }\end{array}$ & $\begin{array}{c}\text { Interactúa usualmente } \\
\text { con docentes y } \\
\text { estudiantes }\end{array}$ & $\begin{array}{l}\text { No dispone de pautas para } \\
\text { acceso ni uso adecuado de } \\
\text { información } \\
\text { Depende de las } \\
\text { indicaciones del docente } \\
\text { para el uso de software }\end{array}$ \\
\hline Tipo 6 & $\begin{array}{l}\text { Busca información de } \\
\text { manera adecuada e } \\
\text { inadecuada } \\
\text { Selecciona información } \\
\text { hallada en algunos casos, } \\
\text { y no en otros, para } \\
\text { responder lo pedido }\end{array}$ & $\begin{array}{l}\text { Espera indicación del } \\
\text { docente para usar } \\
\text { software } \\
\text { No tiene criterios para } \\
\text { seleccionar software y no } \\
\text { hace un uso adecuado }\end{array}$ & $\begin{array}{l}\text { Interactúa con } \\
\text { compañeros usualmente } \\
\text { La única forma de } \\
\text { aprender matemática que } \\
\text { reconoce es a través de la } \\
\text { explicación del docente }\end{array}$ & $\begin{array}{l}\text { Es irregular en el acceso y } \\
\text { uso de información } \\
\text { Depende de las } \\
\text { indicaciones del docente } \\
\text { para el uso de software } \\
\text { Concibe como una única } \\
\text { forma de aprender } \\
\text { matemática la explicación } \\
\text { del docente }\end{array}$ \\
\hline
\end{tabular}

Fuente: Elaboración propia, 2021. 
En esta síntesis quedan de manifiesto aspectos que los estudiantes, que se asocien con cada tipo, tendrían que seguir desarrollando. Particularmente, ponemos atención a la escasa autonomía que se refleja y que ratifica la necesidad de fortalecer el desarrollo de habilidades en los estudiantes, desde el inicio de los estudios superiores.

\section{A modo de cierre}

Retomamos aquí la problemática que nos llevó a plantear y realizar esta investigación. Y, a propósito de ella, queremos enfatizar lo que expresa el Programa de Acceso y Acompañamiento a Estudiantes de Grado y Pregrado de la UNGS:

Se señala entonces que las instituciones deben asumir el desafío de no soslayar esta problemática, concentrando esfuerzos en mejorar las experiencias de los estudiantes particularmente a partir de su ingreso y durante el primer año de las carreras. Esto comporta el desarrollo de estrategias que favorezcan el aprendizaje de su "oficio de estudiante universitario", entendiendo que la mayor parte de los saberes y competencias allí implicados no constituyen el punto de partida de esta etapa sino un punto de llegada intermedio respecto del desarrollo total del proceso formativo. (UNGS, 2018, p. 8, comillas del autor)

En este marco, desde el Taller de Matemática se pretende contemplar las necesidades específicas de los estudiantes que se acercan a la Universidad. En este sentido, conocer la perspectiva de los estudiantes respecto a la habilidad matemática estudiada, resulta un insumo valioso. Nos permite conocer cómo se perciben respecto de ella, a la vez que quedan de manifiesto sus imágenes culturales o concepciones sobre la enseñanza, el aprendizaje y sobre la matemática. A partir de este estudio, hemos encontrado enfatizada la visión de aprender matemática asociada a recibir explicaciones del docente, en muchos casos reforzada por una actitud pasiva, expresada en esperar indicaciones de uso de software, de cuándo interactuar y con quién, etcétera. Esto, a su vez, se combina con una imagen de la matemática negativa asociada a una concepción absolutista de la matemática (ERNEST, 2000) que suele estar presente en los estudiantes. 
Si, tal como señala Ernest (2000, p. 2), “Bajo ese punto de vista las matemáticas son consideradas como un cuerpo de sabiduría objetivo, absoluto, cierto e inmutable, que se apoya en las bases firmes de la lógica deductiva", no sorprende, entonces que los estudiantes consideren que los resultados que ofrece el software no estén en discusión, deban ser matemáticamente correctos o que la exploración con software específicos no sea parte del quehacer matemático que asumen.

Reconocemos las limitaciones de este estudio, como ocurre en toda investigación educativa. Al respecto señalamos el alcance circunscripto a un único grupo de estudiantes y al número de respuestas obtenidas, a raíz de no haber impuesto la obligatoriedad de completar el cuestionario. Tomamos esta decisión para evitar respuestas dadas por obligación, seleccionando opciones sin que quede expresada la real perspectiva de los estudiantes. Asimismo, la tipología presentada emerge de este grupo de estudiantes, por lo que no tiene pretensión de generalidad. Mencionamos también que nos queda ajustar algunas de las frases del cuestionario para evitar a futuro, el uso de palabras como únicamente o espontáneamente que podrían haber complicado la comprensión, y por lo tanto las respuestas. Por ejemplo "interactúo con el docente únicamente cuando me lo indica" o "uso el software únicamente para: verificar, hacer cuentas o graficar más prolijo". Por otra parte, queda por delante indagar con los estudiantes lo que subyace a las respuestas “ni de acuerdo ni en desacuerdo". Interpretamos que esa elección podría darse en casos en los que el asunto clave de la frase no le ha ocasionado al estudiante producir respuestas matemáticamente erróneas. O bien, si ocurrió, le pasó inadvertido el motivo. Por ejemplo: ante la frase "el software siempre produce/ofrece respuestas/resultados correctos", un estudiante que responde "ni de acuerdo ni en desacuerdo", probablemente no se encontró, aún, con respuestas erróneas de un software. Si esto hubiera ocurrido y el alumno traslada esas respuestas a una entrega y el docente le hace ver lo erróneo, podría ocurrir o bien que se dé cuenta del cuidado que debe ponerse en el uso de software (y la elección al grado de acuerdo cambiaría sustantivamente) o que no se dé cuenta que la causa está en el recurso y su uso. Para refinar el estudio en este sentido necesitaremos entrevistar estudiantes. 
Señalamos, también, que consideramos valioso como transferencia -de estos resultados parciales- al plano docente, la tipología propuesta para desempeños de estudiantes en esta habilidad, dado que permite tener claridad en qué cuestiones se deben trabajar para favorecer el avance en estos desempeños.

Nuestro trabajo de investigación continúa con la evaluación, desde la perspectiva docente, del desarrollo de la habilidad estudiada en estudiantes del TIO-Matemática. Estamos transitando esa etapa, la que nos permitirá contrastar y poner en diálogo las miradas desde el estudiante y desde el docente respecto al acceso y uso de nuevas tecnologías para aprender matemática. 


\section{Referencias}

BRAVO, Daniel. Nuevas tecnologías en la enseñanza y aprendizaje de la matemática en el Colegio Del Castillo: descripción del uso actual y propuesta de actualización tecnológica. Tesis (Licenciatura en Tecnología Educativa) - Universidad Tecnológica Nacional, Buenos Aires, 2016. Disponible en:

https://www.researchgate.net/publication/339182469_Nuevas_tecnologias_en_la_ensen anza_y_aprendizaje_de_la_matematica_en_el_Colegio_Del_Castillo_descripcion_del_uso _actual_y_propuesta_de_actualizacion_tecnologica. Acceso en: 1 abr. 2021.

CARNELLI, Gustavo. Matemática en el ingreso a las universidades nacionales argentinas: análisis de propuestas de actividades de aprendizaje. Tesis (Doctorado de la Universidad de Palermo) - Facultad de Ciencias Sociales, Universidad de Palermo, Buenos Aires, 2014. Disponible en: https://www.researchgate.net/publication/341272571_Carnelli-

TesisDoctoral. Acceso en: 1 abr. 2021.

COBO ROMANÍ, Juan. El concepto de tecnologías de la información. Benchmarking sobre las definiciones de las TIC en la sociedad del conocimiento. Zer. Revista de Estudios de Comunicación, Barcelona, v. 14, n. 27, p. 295-318, 2009. Disponible en:

https://ojs.ehu.eus/index.php/Zer/article/view/2636/2182. Acceso en: 1 abr. 2021.

ERNEST, Paul. Los valores y la imagen de las matemáticas: una perspectiva filosófica. Uno. Revista de Didáctica de las Matemáticas, Barcelona, n. 23, p. 9-28, 2000.

POCHULU, Marcel; RODRÍGUEZ, Mabel (comps.). Educación matemática: aportes a la formación docente desde distintos enfoques teóricos. Los Polvorines: Universidad Nacional de General Sarmiento, Villa María: Editorial Universitaria de Villa María, 2015.

RODRÍGUEZ, Mabel. Habilidades matemáticas: una aproximación teórica. Educação Matemática Pesquisa, São Paulo, v. 18, n. 2, p. 809-824, 2016. Disponible en: http://revistas.pucsp.br/index.php/emp/article/view/26016/pdf. Acceso en: 1 abr. 2021.

UNIVERSIDAD NACIONAL DE GENERAL SARMIENTO (UNGS). Programa de Acceso y Acompañamiento a Estudiantes de Grado y Pregrado. Los Polvorines: Documento interno, 2018.

Recebido em: 11/04/2021 Aprovado em: 08/05/2021

Universidade do Estado de Santa Catarina - UDESC Programa de Pós-Graduação em Educação - PPGE 
Revista Linhas

Volume 22 - Número 49 - Ano 2021

revistalinhas@gmail.com

Mabel Alicia Rodríguez - Víctor Hugo González - Doris Rodríguez

Acceso y uso de las nuevas tecnologías para aprender matemática desde la perspectiva del estudiante 